\title{
Muskelmassen und Akneblüte
}

\begin{abstract}
Wenn Bodybuilder ihre Muskulatur mit Anabolika zum Wachstum pushen, riskieren sie erhebliche Nebenwirkungen, von der Akne bis zu Leberschäden. Trotzdem ist der Missbrauch dieser Präparate häufig. Einer Befragung in schleswig-holsteinischen Fitnessstudios zufolge hat jeder vierte männliche Besucher schon einmal versucht, seine Trainingserfolge damit zu beschleunigen.
\end{abstract}

— Beliebt sind Testosteron und synthetische Derivate davon (anabol-androgene Steroide, AAS). Doch die haben auch Nachteile: Während unter der Haut die Muskeln wachsen, sprießen darüber oft Pickel. Letzteres führt den Patienten dann zum Arzt. Der sollte hellhörig werden, wenn ihm neben der Akne auch ausgeprägte Muskelmassen auffallen, so Prof. Martin Schaller, Tübingen: „Wenn die Akne zudem nur schwer zu behandeln ist, sollte man an eine Bodybuilder-Akne denken."

Generell sorgen AAS dafür, dass die Haut fettiger wird. Das kann zur Seborrhö führen und selbst bei Patienten, die solche Probleme bislang nicht kannten, Akne auslösen. Sie ist das auffälligste Zeichen des Missbrauchs. Eine bestehende Akne kann sich zudem unter AAS-Einfluss verschlimmern. Dabei können schwerste Veränderungen entstehen - bis hin zu eingebluteten Knoten oder einer Akne fulminans mit Gelenkbeschwerden und Fieber.

\section{Männlichkeit geht flöten}

Gleichzeitig geht die hart erarbeitete männliche Ausstrahlung flöten: Der muskelbepackte Körper verweiblicht.
Weil er als Reaktion auf die Zufuhr von außen die Eigenproduktion an Testosteron und dessen Vorläufern herunterfährt, schrumpfen die Hoden, die Potenz nimmt ab und die Fruchtbarkeit schwindet. Außerdem wächst die Oberweite: Man(n) bekommt Busen.

Schaller empfiehlt, Patienten bei entsprechendem Verdacht direkt nach der Einnahme von Anabolika zu fragen. Denn da die Nebenwirkungen sie oft stark belasten, sind viele durchaus einsichtig. Dann ist die Therapie einfach: Der Patient wird über die Anabolikarisiken aufgeklärt und setzt die Präparate ab. Eine schwere Akne behandelt Schaller anschließend mit niedrig dosiertem Isotretinoin (10-20 mg/dl): dass sie nicht wiederkommt."

\section{Hohe Androgenspiegel bei Frauen}

Im Gegensatz zu Bodybuildern leiden Frauen mit polyzystischem Ovarsyndrom (PCOS) wider Willen unter zu hohen Androgenspiegeln. Dieses Übermaß wird bei PCOS-Frauen von $\mathrm{Ne}$ bennierenrinden und Ovarien erzeugt. Dabei sind ihre Ovarien oft polyzystisch und eine Menstruation haben sie gar nicht oder in reduzierter Frequenz. „Damit haben Sie die beste Chance,
Unter den sichtbaren Symptomen leiden die Patientinnen meist sehr. So kann sich der Überschuss an Androgenen durch Akne bemerkbar machen. Sie bleibt auch der erwachsenen Frau erhalten und ist meist schwer zu behandeln. Zusätzlich fällt oft ein männliches Behaarungsmuster auf, z. B. Bartwuchs oder eine Haarstraße vom Genitalbereich in Richtung Nabel. Die Kopfhaare dagegen fallen vermehrt aus.

HELGA BRETTSCHNEIDER -

Polyzystisches Ovarialsyndrom

\section{Rezepte gegen Akne} und Frauenbart

In der Therapie das PCOS arbeiten Gynäkologen/Endokrinologen und Dermatologen zusammen. Kontrazeptiva mit antiandrogener Komponente oder niedrig dosiertes Dexamethason verringern die Androgenproduktion. Das bessert Akne und Haarausfall, nach rund drei Monaten sind Effekte sichtbar. Efflonitin wird topisch gegen Hirsutismus angewendet. In der Aknetherapie hat sich Isotretinoin in einer Dosis von 10-20 mg/dl bewährt. Voraussetzung ist, dass die Patientin die Pille nimmt und nicht schwanger ist. Sonst darf nur topisch behandelt werden.

- Derma Update, Frankfurt/Main, 9./10. November 2007 (Veranstalter: med update $\mathrm{GmbH}$ ) 